\title{
PA-046 A MALARIA VACCINE SITE CHARACTERISATION: PREVALENCE AND SPECIES DISTRIBUTION OF PLASMODIUM MALARIA IN A MALARIA ENDEMIC SETTING OF BURKINA FASO (WEST AFRICA)
}

Amidou Diarra, Issa Nebie, Alfred B Tiono, Guillaume Sanou, Alphonse Ouedraogo, Amadou T Konate, Baptiste J Yaro, Issiaka Soulama, Sirima Sodiomon. CNRFP, Burkina Faso

\subsection{6/bmjgh-2016-000260.80}

Background Any development of a vaccine strategy or its implementation is based on a good knowledge of the biology of the pathogen and its temporal and spatial distribution. In the case of malaria, the main four species responsible for the disease though having a similar biological structure are not equally represented spatially due to factors such as environment and susceptibility of human to species.

Methods In order to characterise a site for a future malaria vaccine implementation in terms of malaria prevalence and its species distribution, two cross sectional studies were conducted in October and March corresponding to the high and low malaria transmission season, respectively, in the Banfora Health District. A total of 1203 volunteers aged from 0.5 to 45 years consented to participate in the study. During each survey, after a brief physical examination, blood was taken from each volunteer by finger prick to perform thick and thin blood film examination. Blood smears collected were air dry and the thin film fixed with methanol. Dry smears were then stained with controlled PH Giemsa buffer solution and checked for malaria parasite using light microscope.

Results Malaria prevalence was markedly high during high malaria transmission: $54.26 \%$ compared to low malaria transmission season $39,40 \%$. Plasmodium index was $46.8 \%$ with a gametocyte index of $11 \%$. Main species present in the study area were $P$. falciparum, $P$. malariae and $P$. ovale. Species distribution was almost the same across the two seasons with P. falciparum being more prevalent compared to other species with respectively $97.8 \%$ and $98.7 \%$.

Conclusions $P$. falciparum, $P$. malariae and $P$. ovale are the three main species in the study area with almost the same distribution across the two seasons. 